\title{
AVALIAÇÃO DE GENÓTIPOS DE TRIGO NO VALE DO PARANAPANEMA (SP) DE 1985 A $1991\left({ }^{1}\right)$
}

\author{
JoĀo CARLOS FELÍCIO $\left({ }^{2}\right)$, CARLOS EDUARDO DE OLIVEIRA CAMARGO $\left({ }^{2,4}\right)$ \\ e MÁRIO JOSÉ PEDRO JÚNIOR $\left({ }^{3,4}\right.$ )
}

\begin{abstract}
RESUMO
Estudou-se, durante o período 1985-91, o comportamento de 21 cultivares de trigo no Vale do Paranapanema, Sudoeste paulista, avaliando-se a produtividade de grãos, as reaçðes aos agentes causais de ferrugem-da-folha, da helmintosporiose $\mathrm{e}$ do oídio, e às condiçðes climáticas da região. A precipitação pluvial foi dividida em decêndios dentro de cada mês (março a outubro). $O$ excesso de umidadè no segundo decêndio de junho de 1987 favoreceu a ocorrência das moléstias estudadas. Os cultivares OCEPAR 14, IAC 227, IAPAR 17, IAC 60, IAC 28, IAC 18, IAC 17 e IAC 21 apresentaram produtividade média superior à do 'BH 1146' (testemunha), e os cultivares OCEPAR 7 e Alondra foram os menos produtivos. A ocorrência da mancha foliar do trigo, causada por Cochliobolus sativus, foi generalizada no periodo, aumentando em intensidade com a elevação do indice pluvial, e a da ferrugem-da-folha e do oídio, muito variável e com baixa freqüência.
\end{abstract}

Termos de indexação: trigo, cultivares, resistência às moléstias, produção de grãos, indice pluvial.

\section{ABSTRACT \\ EVALUATION OF WHEAT GENOTYPES FOR THE PARANAPANEMA VALLEY, STATE OF SÃO PAULO, BRAZIL, FROM 1985 TO 1991}

Twenty one wheat cultivars were evaluated in trials carried out at Paranapanema Valley Region, Southwest of the State of São Paulo, Brazil, taking into account the grain yield and the reactions to the causal agents of leaf spot, leaf rust and mildew. The amount of rainfall was divided in periods of ten days in each month (March to October). The rain excess occurred during the second period of ten days on June, 1987, increased the occurrence of the evaluated diseases. The cultivars OCEPAR 14, IAC 227, IAPAR 17, IAC 60, IAC 28, IAC 18, IAC 17 and IAC 21 showed higher grain yields than the 'BH $1146^{\prime}$ used as control, and the cultivars OCEPAR 7 and Alondra presented the lowest yields. The leaf spot, caused by Cochliobolus sativus exhibited generalized occurrence in the evaluated trials increasing in intensity as the amount of rainfall increased. The occurrence of leaf rust and mildew was variable and presented low frequency during the period.

Index terms: wheat, cultivars, disease resistance, grain yield, rainfall index.

(1) Recebido para publicação em 10 de novembro de 1992 e aceito em 19 de abril de 1993.

$\left({ }^{2}\right)$ Seção de Arroz e Cereais de Inverno, Instituto Agronômico (IAC), Caixa Postal 28, 13001-970 Campinas (SP).

$\left({ }^{3}\right)$ Seção de Climatologia Agrícola, IAC.

(') Com bolsa do CNPq. 


\section{INTRODUÇÃO}

Essencialmente, há duas maneiras para elevar a produção mundial de alimentos: pelo aumento de produtividade ou pela ocupação de novas áreas. Ambas são importantes, porém a maior contribuição deverá ser obtida mediante a segunda, uma vez que a produtividade nos principais paises produtores de alimento (Estados Unidos, Canadá, França, Argentina e Austrália) já atingiu niveis bastante altos (Goedert et al., 1980).

A variedade de trigo Frontana foi extensivamente cultivada por sua tolerância a solos ácidos e resistência à raça 15 da ferrugem-do-colmo. Em 1963, os técnicos do trigo lançaram a 'IAS 20', cuja origem em experimentos foi por volta de 1960. Essa variedade foi gradativamente substituindo a Frontana, que, sendo suscetivel à raça 17 (e à 11 também) de ferrugem-do-colmo, perdeu importância em área semeada. Posteriormente, a 'IAS 50' veio substituir, no Rio Grande do Sul, a 'IAS 20' (Duarte, 1974).

A continuidade da estabilidade da produção, ao longo dos anos, segundo Mundstock (1983), deve-se à contínua disponibilidade de novos genótipos. As variedades de trigo permanecem em cultivo, em média, geralmente por cinco anos, obrigando continuamente os programas de melhoramento a dispor de novos genótipos, bem adaptados e resistentes às principais moléstias, em substituição aos que apresentam redução na produtividade no decorrer dos anos.

No Estado de São Paulo, de acordo com Cuyabano (1964), as variedades em cultivo demonstraram alta rusticidade, e o solo onde eram cultivadas nem sempre recebia, por parte do agricultor, atenção quanto à escolha e ao preparo. A semeadura do trigo por anos sucessivos no mesmo terreno tem sua produção diminuída, em vista do aumento do potencial de inóculo de patógenos causadores de moléstias, como helmintosporiose, oídio e septoriose, patógenos esses cujos esporos ficam no terreno junto aos restos de cultura.

Para os agricultores do Paraná e do Rio Grande do Sul, principalmente, a cultura do trigo tem elevada expressão econômica. Esse produto ocupou, em 1986, quase um quarto da área cultivada no Paraná e um sexto no Rio Grande do Sul. Em São Paulo, somente nos últimos anos, com os estímulos do Governo Federal aliados a novos desenvolvimentos tecnológicos, o trigo vem ampliando sua área, atingindo $4,4 \%$ da área agricultável do Estado. Isso é bastante expressivo, pois a triticultura tem grande concorrência de outras culturas, dificultando, portanto, sua expansão (Junqueira \& Silva, 1988).

Para ampliar ainda mais a área semeada e a produtividade da triticultura no Estado, há necessidade de novos cultivares, com maior produtividade, adaptados às regiôes com ou sem irrigação, resistentes às moléstias, tolerantes à acidez do solo e eficientes na utilização de nutrientes, visando ao aumento dos lucros para os triticultores (Camargo \& Felício, 1986), via diminuição dos custos de produção pela economia dos insumos agrícolas.

\section{MATERIAL E MÉTODOS}

Foram desenvolvidos, no período de 1985-91, no Estado de São Paulo, experimentos com a cultura de trigo no Vale do Paranapanema - localidades de Cândido Mota, Cruzália, Maracaí e São José das Laranjeiras - com o objetivo de verificar, em condições de sequeiro, o comportamento do trigo.

Nesses ensaios, avaliaram-se os cultivares Alondra, Anahuac, IAC 5, IAC 17, IAC 18, IAC 21, IAC 23, IAC 24, Paraguai 281, PAT 72247, IAC 28, IAC 162, OCEPAR 7, OCEPAR 11, IAPAR 17, IAC 72, IAC 25, IAC 60, OCEPAR 14 e IAC 227 (com, no mínimo, três anos em experimentação consecutiva), os quais foram comparados com o BH 1146, utilizando-se o método de análise conjunta de experimentos com tratamento comum (Pimentel Gomes, 1970).

Os ensaios foram compostos de seis linhas de $3 \mathrm{~m}$ de comprimento, separadas de $0,20 \mathrm{~m}$, e um espaçamento lateral entre as parcelas de $0,60 \mathrm{~m}$. A densidade de semeadura foi na base de 350 sementes por metro quadrado de área semeada. Na colheita, tomaram-se as seis linhas 
de cada parcela, com área útil de $3,6 \mathrm{~m}^{2}$, e empregou-se o delineamento estatístico de blocos ao acaso, com quatro repetições por local.

A adubação foi aplicada a lanço, de acordo com a análise do solo, usando-se fórmulas compostas de acordo com a recomendação específica para cada local, com base nas tabelas de adubação e calagem do Instituto Agronômico (Raij et al., 1985).

Os dados termopluviométricos diários relativos ao período considerado foram obtidos do Posto Meteorológico da Fazenda Canadá (lat. $22^{\circ} 40^{\prime}$ S.; long. $50^{\circ} 25^{\prime} \mathrm{W}$.; alt. $569 \mathrm{~m}$ ), no município de Assis (SP), mantido em colaboração com o IAC, podendo ser considerado como representativo de toda a região estudada.

Para avaliar o comportamento dos cultivares com relação às principais moléstias em condições naturais de infecção, empregaram-se escalas diafragmáticas de leitura. Para a ferrugem-da-folha (Puccinia recondita Rob. ex Desm. f. sp. tritici Eriks), a avaliação foi realizada em plantas adultas pela escala modificada de Cobb, empregada por Schramm et al. (1974), composta por números, estimando a porcentagem de ataque da moléstia na folha.

Para a mancha foliar - causada pelo fungo Helminthosporium sativum sp. ou $H$. tritici repen- tis - a severidade foi estimada em porcentagem de área foliar infectada (0-99\%), observando-se as folhas superiores das plantas, de acordo com o método proposto por Mehta (1978).

A incidência de oídio (Erysiphe graminis f. sp. tritici foi avaliada desde o início do perfilhamento até o espigamento pleno, sob infecção natural de campo. O coefíciente médio de infecção (CMdI) foi estimado em porcentagem de área foliar infectada de $0-99 \%$, de acordo com o método utilizado por Linhares (1979).

\section{RESULTADOS E DISCUSSÃO}

A triticultura está sujeita às oscilações climáticas devido ao seu período de cultivo (outono-inverno). Anos com baixa produtividade estão associados a estiagens prolongadas, a excesso de chuvas ou a geadas. Anos com elevada produtividade estão associados a boas condiçðes climáticas.

A precipitação pluvial dividida em decêndios em março-outubro para o período de 1985-91, está caracterizada na figura 1 para $o$ primeiro decêndio; na figura 2 para o segundo e na figura 3 para o terceiro decêndio de cada mês em estudo.

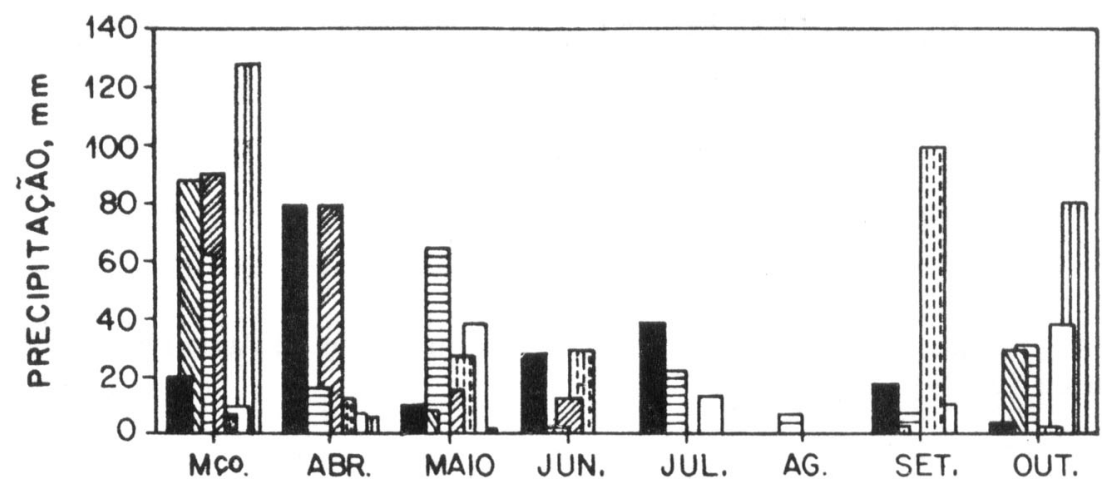

MESES

$\begin{array}{llll}1985 & 1986 & 1987 & \\ 1989 & \square & & 1990\end{array}$

Figura 1. Precipitação pluvial ocorrida no primeiro decêndio na região de Assis (Vale do Paranapanema) entre março e outubro de 1985-91. 


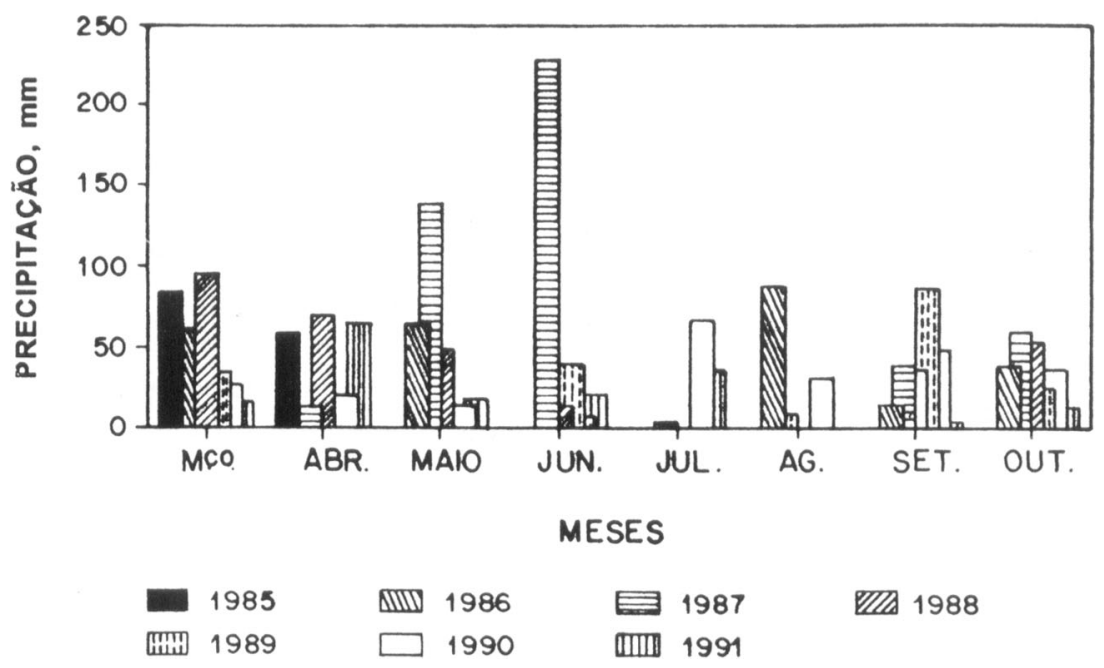

Figura 2. Precipitação pluvial ocorrida no segundo decêndio na região de Assis (Vale do Paranapanema) entre março e outubro de 1985-91.

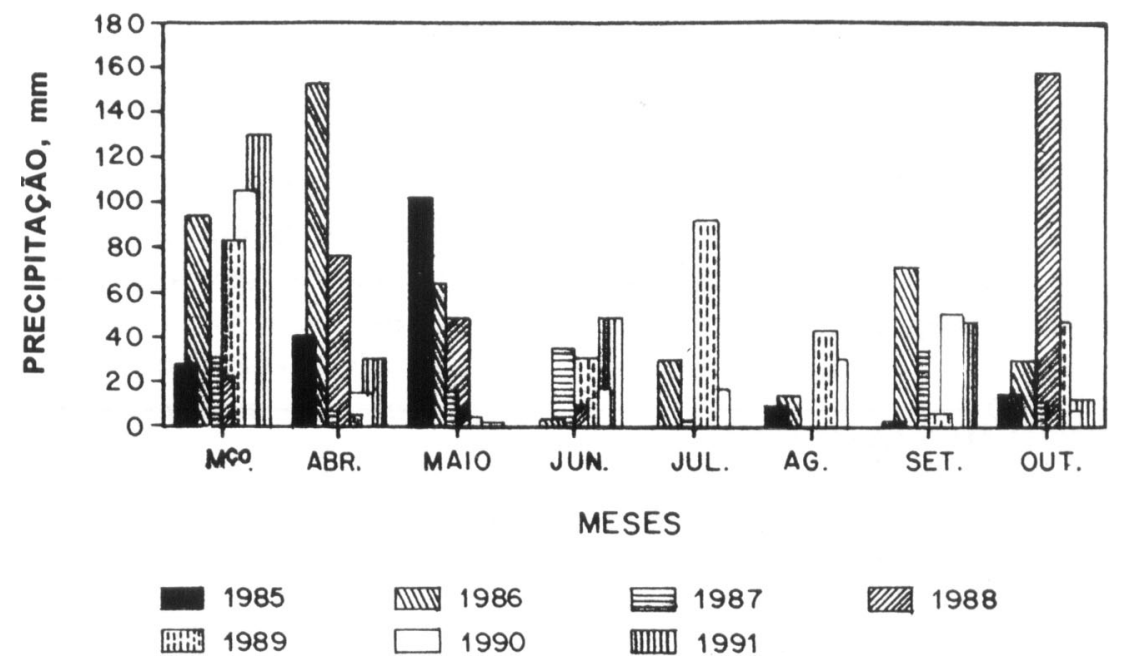

Figura 3. Precipitação pluvial ocorrida no terceiro decêndio na região de Assis (Vale do Paranapanema) entre março e outubro de 1985-91.

A análise dos dados apresentou uma tendência de precipitaçðes mais freqüentes no segundo decêndio (Figura 2) de cada mês, independentemente do ano em questão, exceto em março, cuja tendência foi a concentração das chuvas no terceiro decêndio, quando boas precipitações são impor- tantes, pois representam reserva hídrica para a cultura. De acordo com Felicio et al. (1991), independente do cultivar semeado, o terceiro decêndio de março e o primeiro de abril foram as melhores épocas para a semeadura do trigo no Vale do Paranapanema (SP). 
O excesso de umidade no segundo decêndio de junho de 1987 (228 mm) favoreceu a ocorrência de moléstias, como relatado por Felicio et al. (1986). Junho e agosto, principalmente este último, tendem a apresentar baixas precipitações, favorecendo a maturação e a colheita. A ocorrência de chuvas no terceiro decêndio de agosto e no primeiro e segundo de setembro, além de dificultar a colheita, deprecia a qualidade final do produto, ocasionando baixo peso hectolítrico e provocando excesso da atividade enzimática nos grãos, o que resulta em farinhas de baixa qualidade industrial.

Os quadrados médios das análises da variância para as causas da variação cultivar $\mathrm{x}$ ano $e$ ano $x$ cultivar para rendimentos de grãos, nos ensaios instalados nas localidades de Cruzália,
São José das Laranjeiras, Maracai e Cândido Mota, em 1985-91, encontram-se no quadro 1.

A interação anos $x$ cultivar somente não foi significativa para o cultivar PAT $72247 \mathrm{em}$ São José das Laranjeiras e Cândido Mota, indicando que não houve variação na produção de grãos no período avaliado. Para os demais cultivares em estudo, houve variações na produtividade de acordo com $o$ ano.

A interação cultivares $x$ ano foi não significativa em 1990 e 1991 em Cândido Mota devido às condições climáticas adversas para todos os cultivares nesse biênio. Em 1990, ocorreram geadas em julho e precipitações excessivas por ocasião da colheita (agosto-setembro) e em 1991, deficiência hídrica após a germinação e no final da maturação, afetando os cultivares indistintamente.

Quadro 1. Quadrados médios da análise da variância para rendimento de grãos nos ensaios instalados em Cruzália, São José das Laranjeiras (S.J.L.), Maracaí e Cândido Mota, no Vale do Paranapanema, em 1985-91

\begin{tabular}{clllll}
$\begin{array}{c}\text { Causas } \\
\text { de variação }\end{array}$ & GL & Cruzália & S.J.L. & Maracai & Cândido Mota \\
\hline
\end{tabular}

Cul/1985
Cu1/1986
Cul/1987

$\mathrm{kg} / \mathrm{ha}$

$\begin{array}{lrrrr}18 & 1.903 .338^{*} & 330.604^{*} & 191.043^{*} & 333.305^{*} \\ 20 & 471.410^{*} & 359.183^{*} & 618.656^{*} & 292.104^{*} \\ 20 & 407.004^{*} & 371.027^{*} & 361.507^{*} & 291.753^{*} \\ 16 & 911.302^{*} & 731.692^{*} & 999.368^{*} & 820.202^{*} \\ 13 & 203.415^{*} & 729.869^{*} & 187.459^{*} & 801.020^{*} \\ 11 & 319.220^{*} & 805.959^{*} & 289.210^{*} & 157.340 \mathrm{~ns} \\ 12 & 179.207^{*} & 476.028^{*} & 382.905^{*} & 121.137 \mathrm{~ns} \\ 2 & 1.303 .549^{*} & 1.159 .803^{*} & 3.878 .937^{*} & 1.797 .163^{*} \\ 6 & 1.023 .707^{*} & 856.199^{*} & 1.891 .545^{*} & 648.057^{*} \\ 6 & 2.589 .077^{*} & 3.047 .960^{*} & 2.471 .515^{*} & 1.477 .729^{*} \\ 6 & 1.119 .706^{*} & 1.485 .874^{*} & 1.982 .572^{*} & 501.500^{*} \\ 3 & 359.306^{*} & 3.070 .682^{*} & 879.580^{*} & 1.163 .977^{*} \\ 6 & 1.987 .539^{*} & 2.631 .466^{*} & 1.804 .800^{*} & 993.824^{*} \\ 6 & 1.644 .059^{*} & 1.874 .855^{*} & 1.814 .343^{*} & 2.085 .827^{*} \\ 2 & 563.744^{*} & 1.710 .003^{*} & 1.962 .831^{*} & 700.622^{*} \\ 6 & 1.420 .074^{*} & 957.698^{*} & 1.358 .045^{*} & 1.475 .885^{*} \\ 2 & 1.934 .136^{*} & 698.181^{*} & 2.651 .886^{*} & 482.610^{*} \\ 2 & 309.553^{*} & 107.297 \mathrm{~ns} & 1.499 .440^{*} & 83.097 \mathrm{~ns} \\ 3 & 543.131^{*} & 769.528^{*} & 3.267 .388^{*} & 1.267 .377^{*}\end{array}$

Continua 
Quadro 1. Conclusão

\begin{tabular}{lrrrrr}
\hline $\begin{array}{l}\text { Causas } \\
\text { de variação }\end{array}$ & GL & Cruzália & S.J.L. & Maracai & Cândido Mota \\
& & & & & \\
& & & & & \\
& & & & & \\
Ano/IAC 162 & 6 & $2.739 .390^{*}$ & $830.956^{*}$ & $1.210 .069^{*}$ & $1.724 .398^{*}$ \\
Ano/OCEPAR 7 & 3 & $2.163 .859^{*}$ & $699.575^{*}$ & $683.204^{*}$ & $630.033^{*}$ \\
Ano/OCEPAR 11 & 4 & $1.609 .798^{*}$ & $1.556 .798^{*}$ & $1.353 .117^{*}$ & $1.254 .791^{*}$ \\
Ano/IAPAR 17 & 6 & $2.368 .081^{*}$ & $628.392^{*}$ & $1.958 .940^{*}$ & $2.483 .899^{*}$ \\
Ano/IAC 72 & 5 & $3.068 .500^{*}$ & $846.293^{*}$ & $1.675 .453^{*}$ & $2.406 .546^{*}$ \\
Ano/IAC 25 & 6 & $3.077 .034^{*}$ & $1.042 .168^{*}$ & $1.176 .937^{*}$ & $1.664 .809^{*}$ \\
Ano/IAC 60 & 6 & $3.025 .197^{*}$ & $914.613^{*}$ & $1.231 .881^{*}$ & $2.051 .608^{*}$ \\
Ano/OCEPAR 14 & 5 & $2.442 .182^{*}$ & $2.412 .634^{*}$ & $3.057 .642^{*}$ & $1.355 .962^{*}$ \\
Ano/IAC 227 & 5 & $3.223 .375^{*}$ & $1.959 .716^{*}$ & $1.902 .359^{*}$ & $4.402 .687^{*}$ \\
Resíduo & 351 & 100.830 & 72.247 & 71.715 & 126.480 \\
\hline
\end{tabular}

* Significativo ao nivel de 5\%; ns: năc significativo.

A produtividade média dos cultivares estudados na região encontram-se no quadro 2 .

Os cultivares OCEPAR 14, IAC 227, IAPAR 17, IAC 60 , IAC 28, IAC 18, IAC 17 e IAC 21 apresentaram produtividade média superior à do 'BH 1146', considerando a média das quatro localidades. A variação na produtividade dos cultivares dentro de cada local em estudo deveu-se principalmente à constituição genética de cada um dos cultivares interagindo com fatores do ambiente. Tais fatores, como a acidez do solo, as precipitações pluviais diferenciadas por região e moléstias ligadas ou não às condições do clima, como a helmintosporiose, a ferrugem-da-folha e o oidio, afetaram os genótipos diferenciadamente. Os cultivares OCEPAR 7 e Alondra apresentaram-se como os menos produtivos, ou seja, respectivamente, 15,9 e $20,5 \%$ inferiores ao 'BH 1146 '.

A mancha foliar, que vem afetando a produtividade dos cultivares de trigo nos últimos anos (Luz, 1982; Felicio et al., 1986), apresentou-se como a mais ativa entre as moléstias estudadas. Todos os cultivares demonstraram suscetibilidade em maior ou menor grau. Os cultivares OCEPAR 7, Alondra, IAC 25, OCEPAR 11, IAC 24, IAPAR 17, IAC 162 e Anahuac foram os mais afetados. Por outro lado, os cultivares BH 1146, OCEPAR 14, IAC 28 e IAC 18 apresentaram os menores índices de suscetibilidade à doença (Quadro 3).

O ano com maior epifitia foi 1988 e com menor, 1985 - Figura 4 - pois, de acordo com Mehta (1978), o seu desenvolvimento é favorecido por precipitaçóes contínuas e temperaturas entre 20 e $28^{\circ} \mathrm{C}$.

A ferrugem-da-folha causada por Puccinia recondita $\mathrm{f}$. sp. tritici foi menos severa quando comparada à helmintosporiose. Em 1987 e 1989 , ocorreram suas maiores incidências e em 1991, as menores. Em 1988, ela não apareceu, provavelmente devido à grande ocorrência de helmintosporiose (Quadro 4). Os cultivares IAC 25, IAC 17, IAC 5, IAC 24 e IAC 72 foram os mais suscetíveis, e o PAT 72247, OCEPAR 11 e IAPAR 17, os mais resistentes ao patógeno.

Os anos de 1989 e 1991 foram os mais favoráveis ao aparecimento de oídio causado por Erysiphe graminis f. sp. tritici, o qual não se registrou em 1987 e 1988. O 'IAPAR 17' foi - mais suscetível, diferindo significativamente dos demais genótipos e os cultivares OCEPAR 11 e IAC 60, os mais resistentes (Quadro 5). 
$\mathrm{Na}$ figura 4, foram comparadas as avaliações das três moléstias que ocorreram com maior frequêencia nos sete anos no Vale do Paranapanema. A mancha foliar do trigo, causada por Cochliobolus sativus (forma imperfeita Helminthosporium sativum sp.) teve ocorrência generalizada.
Os cultivares estudados não demonstraram resistência a seu agente causal, pois segundo Luz (1982) ela é influenciada pelo periodo de umidificação pós-inoculação do fungo. Tanto a ferrugem-da-folha como o oídio tiveram ocorrência muito variável e com baixa freqūência no periodo.

Quadro 2. Produtividade média de grăos de trigo dos cultivares estudados nos ensaios de Cruzália, São José das Laranjeiras (S.J.L.), Maracaí e Cândido Mota, no Estado de São Paulo, no período 1985-91

\begin{tabular}{|c|c|c|c|c|c|c|}
\hline Cultivar & Cruzália & S.J.L. & Maracaí & Cândido Mota & Média & $\begin{array}{l}\text { Porcen- } \\
\left.\text { tagem ( }{ }^{1}\right)\end{array}$ \\
\hline & & & $\mathrm{kg} / \mathrm{ha}$ & & & $\%$ \\
\hline OCEPAR 14 & $2.056 a$ & $2.472 a$ & $1.955 \mathrm{ab}$ & $1.927 \mathrm{a}$ & 2.113 & 117,7 \\
\hline IAC 227 & $1.846 \mathrm{a}-\mathrm{c}$ & $2.063 b c$ & $2.101 \mathrm{a}$ & $1.942 \mathrm{a}$ & 1.988 & 110,6 \\
\hline IAPAR 17 & $1.972 \mathrm{a}-\mathrm{c}$ & $2.252 \mathrm{ab}$ & $1.590 \mathrm{c}-\mathrm{f}$ & $1.931 \mathrm{a}$ & 1.936 & 107,7 \\
\hline IAC 60 & $1.920 \mathrm{ab}$ & $2.138 b c$ & $1.817 \mathrm{ab}$ & $1.855 \mathrm{a}$ & 1.933 & 107,6 \\
\hline IAC 28 & $1.902 \mathrm{a}-\mathrm{c}$ & $2.104 b c$ & $1.717 \mathrm{~b}-\mathrm{e}$ & $1.956 \mathrm{a}$ & 1.870 & 104,1 \\
\hline IAC 18 & $1.746 \mathrm{ab}$ & $1.990 \mathrm{~cd}$ & $1.792 \mathrm{~b}-\mathrm{d}$ & $1.949 a$ & 1.869 & 104,0 \\
\hline IAC 17 & $1.750 \mathrm{ab}$ & $2.013 b-d$ & $1.798 \mathrm{a}-\mathrm{d}$ & $1.897 \mathrm{a}$ & 1.865 & 108,8 \\
\hline IAC 21 & $1.692 \mathrm{~b}-\mathrm{d}$ & $1.929 \mathrm{c}-\mathrm{e}$ & $1.786 \mathrm{~b}-\mathrm{d}$ & $1.899 \mathrm{a}$ & 1.824 & 101,5 \\
\hline IAC 25 & $1.740 \mathrm{a}-\mathrm{d}$ & $1.911 \mathrm{c}-\mathrm{e}$ & $1.760 \mathrm{~b}-\mathrm{d}$ & $1.812 \mathrm{a}$ & 1.806 & 100,0 \\
\hline BH $1146(t)$ & $1.680 \mathrm{~b}-\mathrm{d}$ & $1.895 c-f$ & $1.720 \mathrm{~b}-\mathrm{d}$ & $1.891 \mathrm{a}$ & 1.797 & 100,0 \\
\hline IAC 162 & $1.761 \mathrm{a}-\mathrm{d}$ & $1.959 \mathrm{c}-\mathrm{e}$ & $1.557 \mathrm{c}-\mathrm{g}$ & $1.895 a$ & 1.793 & 99,8 \\
\hline IAC 5 & $1.704 \mathrm{a}-\mathrm{d}$ & $1.871 c-f$ & $1.684 c-e$ & $1.874 a$ & 1.783 & 99,2 \\
\hline IAC 24 & $1.751 \mathrm{a}-\mathrm{d}$ & $1.931 \mathrm{c}-\mathrm{e}$ & $1.648 \mathrm{c}-\mathrm{e}$ & $1.788 \mathrm{a}$ & 1.780 & 99,1 \\
\hline PAT 72247 & $1.655 \mathrm{~b}-\mathrm{d}$ & $1.823 c-f$ & $1.553 \mathrm{c}-\mathrm{g}$ & $2.030 \mathrm{a}$ & 1.765 & 98,5 \\
\hline OCEPAR 11 & $1.457 \mathrm{de}$ & $1.753 \mathrm{de}$ & $1.778 b-d$ & $1.840 \mathrm{a}$ & 1.732 & 96,4 \\
\hline IAC 72 & $1.532 \mathrm{c}-\mathrm{e}$ & $1.859 \mathrm{c}-\mathrm{f}$ & $1.417 \mathrm{e}-\mathrm{h}$ & $1.785 \mathrm{a}$ & 1.673 & 93,1 \\
\hline PARAGUAI 281 & $1.686 \mathrm{a}-\mathrm{d}$ & $1.840 c-f$ & $1.306 \mathrm{f}-\mathrm{h}$ & $1.803 \mathrm{a}$ & 1.659 & 92,3 \\
\hline IAC 23 & $1.526 \mathrm{c}-\mathrm{e}$ & $1.653 \mathrm{ef}$ & $1.468 \mathrm{~d}-\mathrm{h}$ & $1.730 \mathrm{ab}$ & 1.639 & 91,2 \\
\hline ANAHUAC & $1.497 \mathrm{de}$ & $1.979 \mathrm{~cd}$ & $1.166 \mathrm{~h}$ & $1.844 \mathrm{a}$ & 1.622 & 90,3 \\
\hline OCEPAR 7 & $1.618 b-d$ & $1.692 \mathrm{~d}-\mathrm{f}$ & $1.426 \mathrm{e}-\mathrm{h}$ & $1.309 \mathrm{ab}$ & 1.511 & 84,1 \\
\hline ALONDRA & $1.168 \mathrm{e}$ & $1.574 \mathrm{f}$ & $1.253 \mathrm{gh}$ & $1.717 \mathrm{~b}$ & 1.428 & 79,5 \\
\hline F. & $6,19 *$ & $10,63^{*}$ & $15,91 *$ & $2,62^{*}$ & & \\
\hline CV\% & 18,79 & 13,86 & 16,37 & 19,40 & & \\
\hline
\end{tabular}

(') Em relação à testemunha, BH 1146.

Médias seguidas por letras distintas diferem entre si ao nível de $5 \%$ pelo teste de Duncan.

* Significativo a $5 \%$. t: testemunha. 
Quadro 3. Quadrados médios da análise da variância para helmintosporiose e graus médios de infecção (porcentagem de área foliar infectada), em estádio de planta adulta, nos ensaios de cultivares de trigo semeados em 1985-91, nos municípios de Cruzália, Maracaí, Cândido Mota e Såo José das Laranjeiras

\section{Causas}

da variação

G.L. Quadrado médio

Infecção

$\%$

\begin{tabular}{|c|c|c|c|}
\hline Ano/Alondra & 2 & 8,33ns & $34,78 \mathrm{ab}$ \\
\hline Ano/Anahuac & 6 & $211,90 \mathrm{~ns}$ & $27,14 a-d$ \\
\hline Ano/BH 1146 & 6 & $472,61^{*}$ & $19,28 \mathrm{e}$ \\
\hline Ano/IAC 5 & 6 & $122,61 \mathrm{~ns}$ & $21,78 \mathrm{c}-\mathrm{e}$ \\
\hline Ano/IAC 17 & 3 & $439,58 *$ & $21,94 c-e$ \\
\hline Ano/IAC 18 & 6 & $330,95^{*}$ & $20,71 \mathrm{de}$ \\
\hline Ano/ IAC 21 & 6 & $343,15^{*}$ & $23,39 c-e$ \\
\hline Ano/IAC 23 & 2 & $233,33 \mathrm{~ns}$ & $25,62 a-e$ \\
\hline Ano/IAC 24 & 6 & $514,28 *$ & $34,28 \mathrm{ab}$ \\
\hline Ano/Paraguai & 2 & 33,33ns & $25,62 \mathrm{a}-\mathrm{e}$ \\
\hline Ano/PAT 72247 & 2 & $89,58 \mathrm{~ns}$ & $21,03 c-e$ \\
\hline Ano/IAC 28 & 3 & $706,25^{*}$ & $20,69 \mathrm{de}$ \\
\hline Ano/IAC 162 & 6 & $700,00^{*}$ & $30,00 a-c$ \\
\hline Ano/OCEPAR 7 & 3 & $1.216,72^{*}$ & $36,38 \mathbf{a}$ \\
\hline Ano/OCEPAR 11 & 4 & $582,50^{*}$ & $34,61 \mathrm{ab}$ \\
\hline Ano/IAPAR 17 & 6 & $520,23^{*}$ & $30,35 a-c$ \\
\hline Ano/IAC 72 & 4 & $482,50^{*}$ & $25,11 b-e$ \\
\hline Ano/IAC 25 & 6 & $653,57^{*}$ & $34,64 a b$ \\
\hline Ano/IAC 60 & 6 & $657,14^{*}$ & $26,07 a-e$ \\
\hline Ano/OCEPAR 14 & 5 & $167,50 \mathrm{~ns}$ & 19,70de \\
\hline Ano/IAC 227 & 5 & $156,66 \mathrm{~ns}$ & $21,78 c-e$ \\
\hline Cul/1985 & 18 & $169,84 \mathrm{~ns}$ & $17,16 \mathrm{D}$ \\
\hline Cul/1986 & 20 & $191,54 \mathrm{~ns}$ & $22,03 \mathrm{C}$ \\
\hline Cul/1987 & 20 & $241,29 *$ & $28,22 B$ \\
\hline Cul/1988 & 16 & $481,98^{*}$ & $43,02 A$ \\
\hline $\mathrm{Cul} / 1989$ & 11 & $246,96 \mathrm{~ns}$ & $28,64 B$ \\
\hline $\mathrm{Cul} / 1990$ & 11 & $99,24 \mathrm{~ns}$ & $27,33 \mathrm{~B}$ \\
\hline $\mathrm{Cul} / 1991$ & 13 & $183,5 \operatorname{lns}$ & $18,58 \mathrm{CD}$ \\
\hline Residuo & 348 & 151,05 & \\
\hline $\mathbf{F}$ & & $4,69 *$ & \\
\hline CV\% & & 46,50 & \\
\hline
\end{tabular}

- Significativo a $5 \%$; ns $=$ næo signifícativo.

Médias seguidas por letras distintas (minúsculas para comparar cultivar dentro dos anos e maiúsculas para comparar anos) diferem entre si a $5 \%$ de significância pelo teste de Duncan. 
Quadro 4. Quadrados médios da análise da variância para ferrugem-da-folha e graus médios de infecção (porcentagem de área foliar infectada), em estádio de planta adulta, nos ensaios de cultivares de trigo semeados em 1985-91, nos municípios de Cruzália, Maracaí, Cândido Mota e São José das Laranjeiras

\section{Causas}

da variação

da variaça

\begin{tabular}{|c|c|c|c|}
\hline & & & $\%$ \\
\hline Ano/Alondra & 2 & $5,33 \mathrm{~ns}$ & $6,83 a b$ \\
\hline Ano/Anahuac & 5 & $32,54 \mathrm{~ns}$ & $2,79 \mathrm{~cd}$ \\
\hline Ano/BH 1146 & 5 & $448,16^{*}$ & $8,91 a-d$ \\
\hline Ano/IAC 5 & 5 & $33,54 \mathrm{~ns}$ & $11,45 a-c$ \\
\hline Ano/IAC 17 & 2 & $190,75^{*}$ & $13,50 \mathbf{a b}$ \\
\hline Ano/IAC 18 & 5 & $65,54 \mathrm{~ns}$ & $5,45 \mathrm{bc}$ \\
\hline Ano/IAC 21 & 5 & $61,54 \mathrm{~ns}$ & $7,79 a-d$ \\
\hline Ano/IAC 23 & 2 & $220,08^{*}$ & $10,33 a-d$ \\
\hline Ano/IAC 24 & 5 & $198,87^{*}$ & $11,12 a-c$ \\
\hline Ano/Paraguai & 2 & $2,74 \mathrm{~ns}$ & $9,09 a-d$ \\
\hline Ano/PAT 72247 & 2 & $2,08 \mathrm{~ns}$ & $0,41 d$ \\
\hline Ano/IAC 28 & 2 & $706,58 *$ & $10,58 a-d$ \\
\hline Ano/IAC 162 & 5 & $50,56 \mathrm{~ns}$ & $4,16 \mathrm{~cd}$ \\
\hline Ano/OCEPAR 7 & 2 & $36,75 \mathrm{~ns}$ & $2,75 c d$ \\
\hline Ano/OCEPAR 11 & 3 & $3,56 \mathrm{~ns}$ & $1,18 d$ \\
\hline Ano/IAPAR 17 & 5 & $12,96 \mathrm{~ns}$ & $2,33 d$ \\
\hline Ano/IAC 72 & 3 & $110,41 \mathrm{~ns}$ & $10,62 a-c$ \\
\hline Ano/IAC 25 & 5 & $202,50 *$ & $13,75 \mathbf{a}$ \\
\hline Ano/IAC 60 & 5 & $83,10 \mathrm{~ns}$ & $7,25 a-d$ \\
\hline Ano/OCEPAR 14 & 4 & $43,75 \mathrm{~ns}$ & $3,75 \mathrm{~cd}$ \\
\hline Ano/IAC 227 & 4 & $132,17 \mathrm{~ns}$ & $7,70 a-d$ \\
\hline Cull/1985 & 18 & $57,48 \mathrm{~ns}$ & $4,51 B$ \\
\hline $\mathrm{Cul} / 1986$ & 20 & $77,95 \mathrm{~ns}$ & $6,25 \mathrm{~B}$ \\
\hline $\mathrm{Cul} / 1987$ & 20 & $262,36^{*}$ & $11,30 \mathrm{~A}$ \\
\hline Cul/1989 & 13 & $227,86^{*}$ & $10,93 \mathrm{~A}$ \\
\hline $\mathrm{Cul} / 1990$ & 11 & $44,3 \operatorname{lns}$ & $4,53 \mathrm{~B}$ \\
\hline $\mathrm{Cul} / 1991$ & 11 & $44,42 \mathrm{~ns}$ & $4,05 B$ \\
\hline Resíduo & 296 & 55,90 & \\
\hline F. & & $5,19^{*}$ & \\
\hline CV\% & & 107,83 & \\
\hline
\end{tabular}

- Significativo a $5 \%$; ns = não significativo.

Médias seguidas por letras distintas (minúsculas para comparar cultivar dentro dos anos e maiúsculas para comparar anos) diferem entre si a $5 \%$ de significância pelo teste de Duncan. 
Quadro 5. Quadrados médios da análise da variância para oídio e graus médios de infecção (porcentagem de área foliar infectada), em estádio de planta adulta, nos ensaios de cultivares de trigo semeados em 1985-91, nos municipios de Cruzália, Maracaí, Cândido Mota e São José das Laranjeiras

\section{Causas}

da variação

G.L. Quadrado médio

Infecçẩo

$\%$

\begin{tabular}{|c|c|c|c|}
\hline Ano/Alondra & 1 & $612,50^{*}$ & $14,20 \mathrm{~b}$ \\
\hline Ano/Anahuac & 4 & $320,00^{*}$ & $11,00 \mathrm{bc}$ \\
\hline Ano/BH 1146 & 4 & $230,37^{*}$ & $9,50 b-d$ \\
\hline Ano/IAC 5 & 4 & $48,12 n s$ & $4,00 \mathrm{~cd}$ \\
\hline Ano/IAC 17 & 1 & $78,12 \mathrm{~ns}$ & $7,33 \mathrm{~b}-\mathrm{d}$ \\
\hline Ano/IAC 18 & 4 & $116,87 \mathrm{~ns}$ & $6,00 b-d$ \\
\hline Ano/IAC 21 & 4 & $79,37 \mathrm{~ns}$ & $5,25 b-d$ \\
\hline Ano/IAC 23 & 1 & $12,50 \mathrm{~ns}$ & $5,45 b-d$ \\
\hline Ano/IAC 24 & 4 & $66,87 \mathrm{~ns}$ & $6,00 b-d$ \\
\hline Ano/Paraguai & 1 & $6,12 \mathrm{~ns}$ & $5,08 b-d$ \\
\hline Ano/PAT 72247 & 1 & $12,50 \mathrm{~ns}$ & $5,45 b-d$ \\
\hline Ano/IAC 28 & 1 & $50,00 \mathrm{~ns}$ & $7,95 b-d$ \\
\hline Ano/IAC 162 & 4 & $240,62 *$ & $10,00 b-d$ \\
\hline Ano/OCEPAR 7 & 1 & $112,50 \mathrm{~ns}$ & $9,20 b-d$ \\
\hline Ano/OCEPAR 11 & 2 & $43,75 \mathrm{~ns}$ & $2,47 d$ \\
\hline Ano/IAPAR 17 & 4 & $505,00 *$ & $24,25 a$ \\
\hline Ano/IAC 72 & 2 & $18,75 n s$ & $3,72 \mathrm{~cd}$ \\
\hline Ano/IAC 25 & 4 & $62,50 \mathrm{~ns}$ & $5,00 \mathrm{~cd}$ \\
\hline Ano/IAC 60 & 4 & $32,50 \mathrm{~ns}$ & $2,75 \mathrm{~cd}$ \\
\hline Ano/OCEPAR 14 & 3 & $164,06 \mathrm{~ns}$ & $8,21 b-d$ \\
\hline Ano/IAC 227 & 3 & $260,41 *$ & $9,15 b-d$ \\
\hline $\mathrm{Cul} / 1985$ & 18 & $87,45 \mathrm{~ns}$ & $6,83 \mathrm{~B}$ \\
\hline $\mathrm{Cul} / 1986$ & 20 & $62,05 \mathrm{~ns}$ & $2,67 \mathrm{C}$ \\
\hline $\mathrm{Cul} / 1989$ & 13 & $440,83 *$ & $13,70 \mathrm{~A}$ \\
\hline $\mathrm{Cul} / 1990$ & 11 & $110,74 \mathrm{~ns}$ & $3,14 \mathrm{C}$ \\
\hline $\mathrm{Cul} / 1991$ & 11 & $153,78^{*}$ & $12,20 \mathrm{~A}$ \\
\hline Resíduo & 234 & 85,03 & \\
\hline F. & & $4,70^{*}$ & \\
\hline CV\% & & 119,53 & \\
\hline
\end{tabular}

- Significativo a 5\%; ns = não significativo.

Médias seguidas por letras distintas (minúsculas para comparar cultivar dentro dos anos e maiúsculas para comparar anos) diferem entre si a $5 \%$ de significância pelo teste de Duncan. 


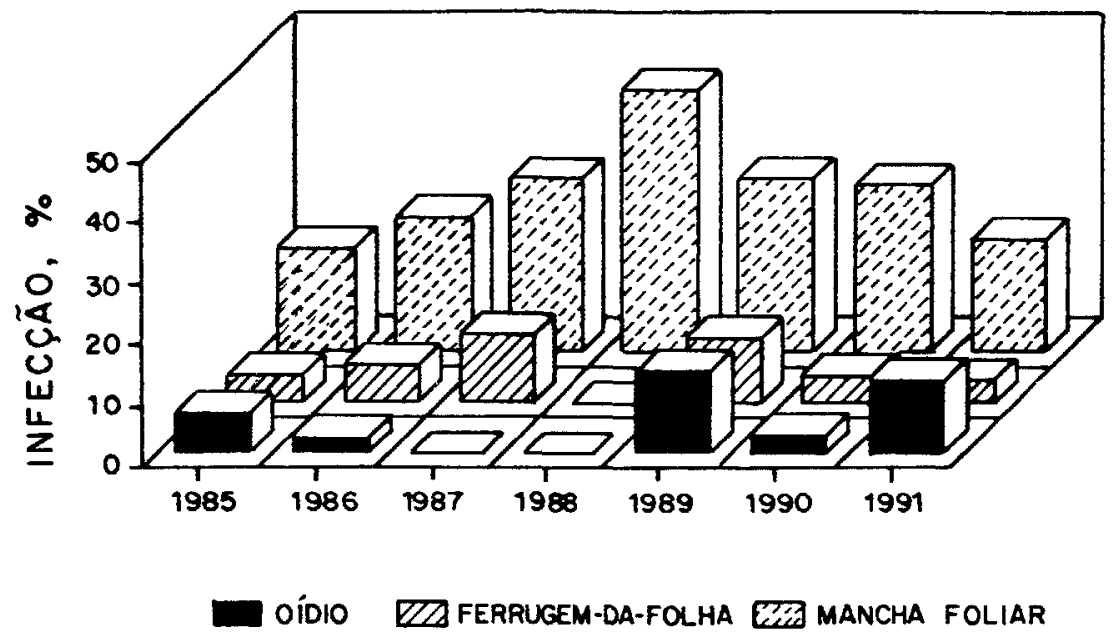

Figura 4. Avaliações comparativas entre as doenças ocorrentes no Vale do Paranapanema em 1985-91

\section{CONCLUSÕES}

1. O regime pluvial foi muito diferenciado de um ano para outro e, conseqüentemente, a produtividade dos cultivares sofreu influência dessa variação climática anual.

2. Os cultivares OCEPAR 14, IAC 227, IAPAR 17, IAC 60, IAC 28, IAC 18, IAC 17 e IAC 21 apresentaram boa adaptação regional, obtendo produtividade superior à da testemunha, BH 1146.

3. A mancha foliar do trigo foi a moléstia de ocorrência generalizada no período, aumentando a sua severidade em função do indice pluvial.

4. A ferrugem-da-folha e o oidio apresentaram-se com severidade muito variável.

\section{REFERÊNCIAS BIBLIOGRÁFICAS}

CAMARGO, C.E. de O. \& FELICIO, J.C. Melhoramento genético do trigo no Estado de São Paulo. O Agronômico, Campinas, 38(3):213-227, 1986.

CUYABANO, N.A. A cultura do trigo. Campinas, Divisão de Assistência Especializada, 1964. 23p. (Instruçð̃es técnicas, 19)
DUARTE, A.R. Tecnologia na agricultura: considerações sobre a produtividade do trigo brasileiro. São Paulo, 1974. 144p. Tese (Doutorado) - USP, 1974.

FELICIO, J.C.; CAMARGO, C.E. de O.; FERREIRA FILHO, A.W.P.; FREITAS, J.G. de \& PEDRO JÚNIOR, M.J. Trigo: épocas de semeadura em Assis (Vale do Paranapanema), SP, no período 1978-82. Bragantia, Campinas, 50(1):115-128, 1991.

FELICIO, J.C.; CAMARGO, C.E. de O.; FREITAS, J.G. de; FERREIRA FILHO, A.W.P.; BARROS, B. de C. \& CAMARGO, M.B.P. de. Avaliação de genótipos de trigo para a região do Vale do Paranapanema no quadriênio 1981-84. Bragantia, Campinas, 45(2):257$277,1986$.

GOEDERT, W.J.; LOBATO, E. \& WAGNER, E. Potencial agrícola da Região dos Cerrados Brasileiros. Pesquisa Agropecuária Brasileira, Brasília, 15(1):1-17, 1980.

JUNQUEIRA, P. de C. \& SILVA, J.R. da. Perspectivas da cultura do trigo na região Centro-Sul. São Paulo, Instituto de Economia Agrícola, 1988. 22p. (Relatório de pesquisa, 22/88)

LINHARES, W.I. Avaliação de cultivares no CNPTrigo quanto ao ataque do míldio (Erysiphe graminis f.sp. tritici) - 1978. In: REUNIÃO DA COMISSÃO NORTE-BRASILEIRA DE TRIGO, 5., Dourados, 1979. Trabalhos apresentados. Passo Fundo, EMBRAPA-CNPT, 1979. p.27-38. 
LUZ, W.C. da. Influência do periodo de umidificação pós-inoculação na reação de cultivares de trigo à mancha foliar (Cochliobolus sativus). In: REUNIÃO NACIONAL DE PESQUISA DE TRIGO, 12., Cascavel, 1982. Resultados de pesquisa. Passo Fundo, EMBRAPA-CNPT, 1982. p.186-191.

MEHTA, Y.R. Doenças do trigo e seu controle. São Paulo, Agronômica Ceres, 1978. 190p. (Ceres, 20)

MUNDSTOCK, C.M. Cultivo dos cereais de estação fria: trigo, aveia, centeio, alpiste e triticale. Porto Alegre, NBS, 1983. 265p.
PIMENTEL-GOMES, F. Curso de estatistica experimental. 4.ed.rev.ampl. Piracicaba, Nobel, 1970. 430p.

RAIJ, B. van; SILVA, N.M. da; BATAGLIA, O.C.; QUAGGIO, J.A.; HIROCE, R.; CANTARELLA, H.; BELLINAZZI JÚNIOR, R.; DECHEN, A.R. \& TRANI, P.E. Recomendaçס̃es de adubaçđo e calagem para o Estado de São Paulo. Campinas, Instituto Agronômico, 1985. 107p. (Boletim técnico, 100)

SCHRAMM, W.; FULCO, W.S.; SOARES, M.H.G. \& ALMEIDA, A.M.P. Resistência de cultivares de trigo em experimentação ou cultivo no Rio Grande do Sul, às principais doenças fúngicas. Agronomia Sulriograndense, Porto Alegre, 10(1):31-52, 1974. 